\title{
Sensitive detection of colorectal cancer in peripheral blood by a novel methylation assay
}

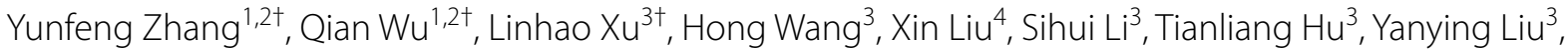
Quanzhou Peng ${ }^{5}$, Zhiwei Chen ${ }^{3,4^{*}}$, Xianrui Wu ${ }^{1,2^{*}}$ and Jian-Bing Fan ${ }^{3,5^{*}}$ (D)

\begin{abstract}
Background: Colorectal cancer (CRC) is a leading cause of cancer-related deaths worldwide. Early detection of CRC can significantly reduce its mortality rate. Current method of CRC diagnosis relies on the invasive endoscopy. Noninvasive assays including fecal occult blood testing (FOBT) and fecal immunological test (FIT) are compromised by low sensitivity and specificity, especially at early stages. Thus, a non-invasive and accurate approach for CRC screening would be highly desirable.
\end{abstract}

Results: A new qPCR-based assay combining the simultaneous detection of the DNA methylation status of ten candidate genes was used to examine plasma samples from 56 normal controls, 6 hyperplastic polys, 9 non-advanced adenomas (NAAs), 22 advanced adenomas (AAs) and 175 CRC patients, using $10 \mathrm{ng}$ of cfDNA. We further built a logistic regression model for CRC diagnosis. We tested ten candidate methylation markers including twist1, vav3-as1, fbn1, c9orf50, sfmbt2, kcnq5, fam72c, itga4, kcnj12 and znf132. All markers showed moderate diagnostic performance with AUCs ranging from 0.726 to 0.815 . Moreover, a 4-marker model, comprised of two previously reported markers (c9orf50 and twist1) and two novel ones (kcnj12 and znf132), demonstrated high performance for detecting colorectal cancer in an independent validation set $(N=69)$ with an overall AUC of 0.911 [95\% confidence interval $(\mathrm{Cl})$ 0.834-0.988], sensitivity of 0.800 [ $95 \% \mathrm{Cl} 0.667-0.933$ ] and specificity of 0.971 [95\% Cl $0.914-1.000]$. The stage-stratified sensitivity of the model was 0.455 [95\% Cl 0.227-0.682], 0.667 [95\% Cl 0.289-1.000], 0.800 [95\% Cl 0.449-1.000], 0.800 [95\% Cl 0.449-1.000] and 0.842 [95\% Cl 0.678-1.000] for advanced adenoma and CRC stage I-IV, respectively.

Conclusion: kcnj12 and znf132 are two novel methylation biomarkers for CRC diagnosis. The 4-marker methylation model provides a new non-invasive choice for CRC screening and interception.

Keywords: Liquid biopsy, Methylation biomarker, Colorectal cancer

*Correspondence: zhiwei_chen@anchordx.com; wuxianr5@mail.sysu.edu.cn; jianbingfan1115@smu.edu.cn

TYunfeng Zhang, Qian Wu and Linhao Xu have contributed equally to this work

${ }^{2}$ Guangdong Provincial Key Laboratory of Colorectal and Pelvic Floor Diseases, The Sixth Affiliated Hospital, Sun Yat-Sen University, Guangzhou 510655, Guangdong, China

${ }^{3}$ AnchorDx Medical Co., Ltd, Unit 502, 3rd Luoxuan Road, International Bio-Island, Guangzhou 510300, China

Full list of author information is available at the end of the article

\section{Introduction}

Colorectal cancer (CRC) is among the three most common cancer types and the second leading cause of cancer related deaths worldwide [1]. Late-stage diagnosis is a major cause of morbidity and mortality of CRC. It is widely accepted that the disease burden can be decreased with proper population-based screening methods, which can detect precancerous lesions and early stage cancer [2]. Currently, CRC screening guidelines recommend colonoscopy and fecal 
immunochemical test (FIT) as the first-tier options [3]. However, due to the invasiveness, dietary restriction requirement and troublesome bowel preparation, colonoscopy has a low compliance rate [4]. The FOBT/ FIT is a non-invasive test which is easy to perform and inexpensive, but it does not have sufficient sensitivity for advanced adenomas (AAs) or stage I CRC detection [4]. Another stool DNA-based colorectal screening test Cologuard was approved by the Food and Drug Administration (FDA) as a colorectal (CRC) screening method for average-risk adults [5]. Furthermore, based on a recent study evaluating the sample preference among screening-aged individuals, $78 \%$ of the survey participants preferred to choose blood test instead of the stool-based FIT [6]. Hence, a non-invasive and accurate blood-based test for early detection of CRC and adenomas could potentially improve patient compliance and is highly desirable.

Aberrant regulation of gene expression by DNA methylation is a well-characterized event in tumorigenesis and is commonly found in tumor suppressor genes among many cancer types, including CRC [7]. Increased levels of circulating-free methylated DNA in the blood of cancer patients have been reported [8]. Indeed, several promising blood-based epigenetic markers with high sensitivity and specificity have been identified in previous studies for the early diagnosis of CRC, but few of them have been validated extensively and commercially available [9-11]. Among them, Epi proColon (blood-based test that examines sept 9 methylation status) is the only blood-based test approved by FDA for CRC screening and has been used clinically for nearly 10 years [12]. However, the value of sept 9 promoter methylation as a CRC screening biomarker has been questioned due to its limited sensitivity, especially for early-stage cancers [13-15]. Owing to the suboptimal performance of commercially available epigenetic tests, discovery and validation of better candidate biomarkers or panels, which are robust in each of the stages of colorectal carcinogenesis from non-advanced adenomas (NAAs) to AAs and then to stages I-IV of CRC, is warranted.

One trend in cancer diagnosis to improve sensitivity is through combination of multiple markers and/or platforms, i.e. multi-omics. For example, it was reported that the sensitivities for CRC detection were 0.718 and 0.505 , for sept 9 methylation and the carcinoembryonic antigen (CEA), respectively. When test results for sept 9 methylation and CEA were combined, the CRC detection rate was increased to $85.7 \%$ [16]. However, the limited amount of blood obtained during a routine test could curb the assay numbers and the potential of maximizing the diagnostic performance, esp. for cell-free DNA (cfDNA) based markers.
Our recent work identified a novel CRC-specific methylation model at tissue level, which was highly sensitive to early-stage CRC and stage-dependent [17]. This model consisted of markers that have been reported previously, e.g. itga4, thbd, vav3-as1 and a few novel ones, including $k c n j 12, z n f 132$, and $s f m b t 2$. In this study, we evaluated the performance of these methylation markers in the plasma cohort, and further refined the methylation model for the application in CRC early detection.

\section{Results \\ Patients and samples}

A total of 272 patients and controls, including 175 CRC patients, 22 patients with advanced adenomas (AAs), 9 patients with non-advanced adenomas (NAAs), 6 patients with hyperplastic polys and 60 healthy controls (Fig. 1) were enrolled in this study from two clinic centers; their plasma samples were used for model development and validation. Of which, 4 samples were excluded from analysis due to hemolysis or inadequate cfDNA amount extracted from plasmas. Relevant clinical characteristics of the cohort including age, gender, histology and tumor staging (AJCC criteria) were listed in Table 1. Among the 268 patients and controls, the percentage of male patients was $46.7 \%$ ( $53.3 \%$, female). The overall median age was 58 years. There were no significant differences of age and gender between non-CRC and CRC groups. Stage I + II cancers comprised $40.0 \%$ of the total CRC patients.

\section{Methylation status of different biomarkers in plasma samples}

The methylation status of 10 candidate markers (twist1, vav3-as1, fbn1, c9orf50, sfmbt2, kcnq5, fam72c, itga4, $k c n j 12, z n f 132)$ were analyzed in all plasma samples $(N=268)$ using a qPCR-based assay (Additional file 1: Figure $\mathrm{S} 1$ and Additional file 1: Table S1), divided into nonCRC group (Normal, HP, NAA) and AA/CRC group (AA and stage I-IV CRC). A percentage of methylated reference (PMR) score was used to measure the methylation levels. After evaluating the methylation level of each marker in $\mathrm{AA} / \mathrm{CRC}$ group and non-CRC group, we found all 10 targeted genes showed significantly higher PMR values in AA/ CRC group compared to non-CRC group, indicating the higher frequency of methylation in tumors (Fig. 2a). The performance characteristics of these markers were shown in Table 2. The area under curves (AUCs) of these markers ranged from 0.726 to 0.815 , with igta 4 achieved the highest AUC value of $0.815, f b n 1$ and $k c n q 5$ showed a mean specificity of $100 \%$ in 2,000 bootstrap samplings. Even though the AUCs of these markers in plasma were lower than what was shown in the tissue [17], all 10 markers had the diagnostic potential for detecting CRC in blood. We 


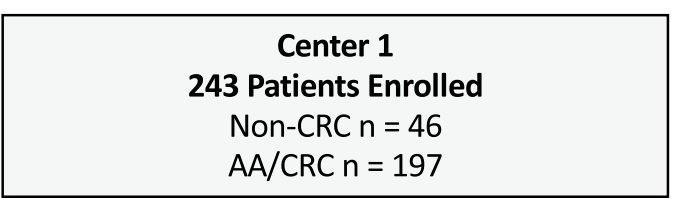

20\% Split

$80 \%$ Split

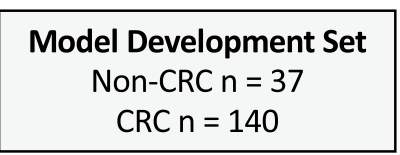

Non-CRC $n=37$

\section{5\% Split}
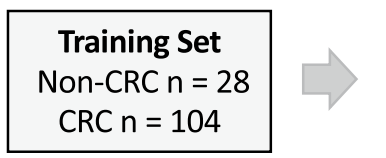

Non-CRC $n=9$

AA/CRC $n=57$

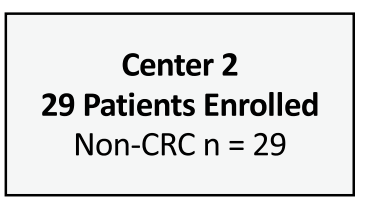

Patients Enrolled

Non-CRC $n=29$

Non-CRC $n=25^{\text {\& }}$

Fig. 1 The study design of development and validation of a cfDNA-based methylation assay for colorectal cancer diagnosis. * advanced precursor cancer lesions only in validation dataset ${ }^{\&} 4$ samples excluded due to hemolysis or or inadequate cfDNA amount extracted from plasmas

Table 1 The demographic and clinical characteristics of the study cohort

\begin{tabular}{|c|c|c|c|c|c|c|}
\hline & \multicolumn{3}{|l|}{ Non-CRC } & \multicolumn{3}{|l|}{$\mathrm{AA} / \mathrm{CRC}$} \\
\hline & Normal & Hyperplastic polys & $\begin{array}{l}\text { Non-advanced } \\
\text { adenomas }\end{array}$ & Advanced adenoma & Tumor & Total \\
\hline Total $(n)$ & 56 & 6 & 9 & 22 & 175 & 268 \\
\hline Age (years) & $46(27-70)$ & $50(38-51)$ & $53(38-76)$ & $57.5(34-86)$ & $60(24-89)$ & $58(24-89)$ \\
\hline \multicolumn{7}{|c|}{ Gender-no. (\%) } \\
\hline Male & 27 & 2 & 2 & 13 & 81 & $125(46.7 \%)$ \\
\hline Female & 29 & 4 & 7 & 9 & 94 & $143(53.3 \%)$ \\
\hline \multicolumn{7}{|l|}{ Stage } \\
\hline Stage I & & & & & $26(14.8 \%)$ & \\
\hline Stage II & & & & & $44(25.2 \%)$ & \\
\hline Stage III & & & & & $21(12.0 \%)$ & \\
\hline Stage IV & & & & & 84 (48.0\%) & \\
\hline
\end{tabular}

also found PMRs of these 10 markers showed a trend of stepwise increase with the progression of CRC (Additional file 1: Figure S2), indicating these markers maybe useful for cancer staging. Furthermore, the analysis of DNA methylation levels of these ten markers suggested that there were strong correlations between kcnq5 and itga4, fam $72 c$ and itga4 (coefficient $>0.800$, Fig. 2b).

\section{Development and validation of the diagnostic model for CRC early detection}

All the plasma samples were divided randomly into two sets with a 4:1 ratio: a model development set (normal
$N=24 ;$ non-advanced-precancerous lesions $N=13$; CRC $N=140$ ) and a validation set (normal $N=32$; non-advanced-precancerous lesions $N=2$; advancedprecancerous lesions $N=22$; CRC $N=35$ ). The model development set was comprised of a training set (normal $N=17$; non-advanced-precancerous lesions $N=11$; CRC $N=104$ ) and a test set (normal $N=7$; nonadvanced-precancerous lesions $N=2$; CRC $N=36$ ) in a way that the age and gender in the testing set is matched to the training set. Due to limited samples, all high-grade intraepithelial adenocarcinoma (HGIN) 


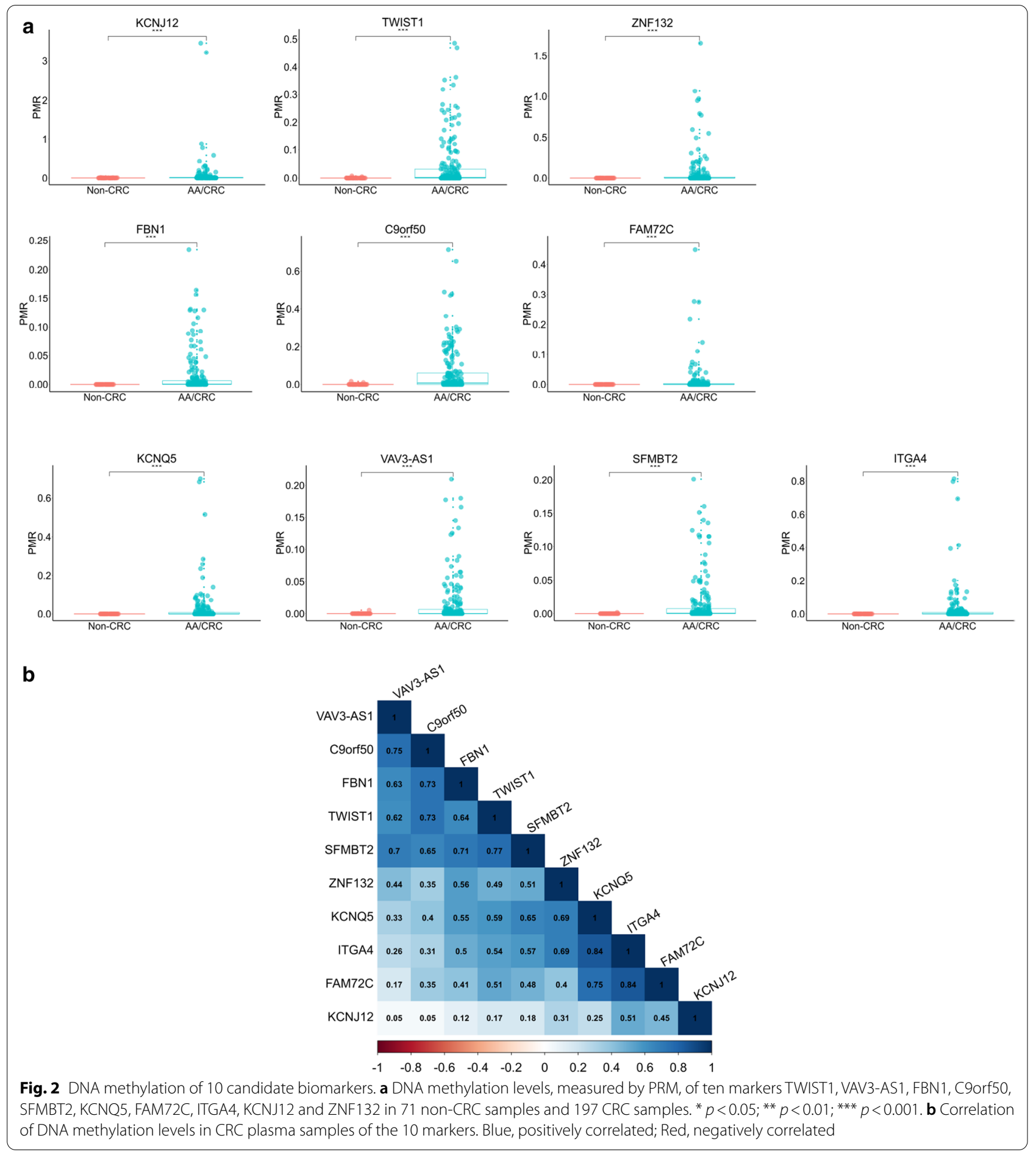

samples, i.e. advanced adenomas, were grouped into the validation set (Additional file 1: Table S2).

We first used all 10 markers to identify the best performing marker combination in 100 splits of the data set using logistic regression, which achieved AUCs of 0.916 [0.863-0.969] and $0.910[0.826-0.995]$ in the training and test sets, respectively (Additional file 1: Figure S3A, B). c9orf50 performance ranked the highest in the 10-marker model regarding the feature coefficient (Additional file 1: Figure $\mathrm{S} 3 \mathrm{C}$ ). A recursive feature elimination process was used to search for all marker combinations that could maintain an AUC within 1\% of the 10-marker model's 
Table 2 The single biomarker performance metrics

\begin{tabular}{llll}
\hline Marker & AUC & Sensitivity & Specificity \\
\hline twist1 & $0.765(0.716-0.815)$ & $0.563(0.497-0.645)$ & $0.972(0.901-1.000)$ \\
vav3-as1 & $0.743(0.705-0.782)$ & $0.503(0.431-0.574)$ & $0.986(0.944-1.000)$ \\
fbn1 & $0.786(0.747-0.826)$ & $0.584(0.508-0.66)$ & $1.000(0.958-1.000)$ \\
c9orf50 & $0.771(0.728-0.814)$ & $0.594(0.523-0.66)$ & $0.972(0.930-1.000)$ \\
sfmbt2 & $0.781(0.744-0.818)$ & $0.574(0.503-0.64)$ & $0.986(0.958-1.000)$ \\
kcnq5 & $0.726(0.689-0.763)$ & $0.467(0.396-0.533)$ & $1.000(1.000-1.000)$ \\
fam72C & $0.785(0.746-0.823)$ & $0.584(0.503-0.66)$ & $0.972(0.915-1.000)$ \\
itga4 & $0.815(0.779-0.851)$ & $0.64(0.569-0.711)$ & $0.986(0.944-1.000)$ \\
kcnj12 & $0.799(0.745-0.853)$ & $0.624(0.457-0.878)$ & $0.887(0.606-0.986)$ \\
znf132 & $0.764(0.718-0.81)$ & $0.563(0.487-0.635)$ & $0.972(0.915-1.000)$ \\
\hline
\end{tabular}

test AUC (0.910) using the smallest number of markers. This resulted in a combination of four genes, $c 9 \operatorname{orf} 50$, kcnj12, znf132 and twist1. As shown in Fig. 3a, b, the AUC in the test set was 0.907 [0.822-0.993], significantly higher than the individual gene models. All four markers in the model had very similar coefficients, indicating the four markers contributing more of less equally to the model performance (Additional file 1: Table S3). The 4-marker model had an overall sensitivity of 0.806 [0.676-0.935] and specificity of 0.889 [0.684-1.000] in the test set, compared to individual markers' sensitivities of 0.594, 0.640, 0.559 and 0.579 and specificities of 0.957 , $0.870,0.957$ and 0.957 for c9orf50, kcnj12, znf132 and twist1, respectively (Table 3 ). To further validate whether the performances of these four markers could be extrapolated to other ethnicities, we calculated the performance of each individual marker on two CRC groups, colon adenocarcinoma (COAD) and rectum adenocarcinoma (READ), comprised mostly of White/Black from TCGA database. As expected, each marker achieved a superior performance in diagnosing CRC with an AUC ranging from 0.642 to 0.962 in cohort COAD and 0.661 to 0.977 in cohort READ, as shown in Additional file 1: Figure S4.

We then tested this 4-marker model in an independent validation set. The overall AUC dropped significantly to 0.796 [0.707-0.886] (Fig. 3c). However, if ruled out the AA samples in the validation set, the AUC increased to 0.911 [0.834-0.988] and was consistent with the diagnostic performance in the test set (Fig. 3d). The sensitivity of the model in AA group was 0.455 [0.227-0.682]. The sensitivity and specificity of the model in the validation set without AA was 0.800 [0.667-0.933] and 0.971 [0.914-1.000], respectively (Table 3 ). These data suggested that the 4-marker model had an enhanced detection sensitivity in detecting all stages CRC compared to individual markers.

\section{Model performance in different stages with CRC progression}

We then evaluated the performance of this model in different stages of CRC. The 4-marker model showed a limited diagnostic performance in detecting AA, with an AUC of 0.614 [0.457-0.770], while achieved an AUC of 0.868 [0.639-1.000], 0.800 [0.408-1.000], 0.935 [0.805-1.000], 0.947 [0.884-1.000] for stage I, II, III and IV, respectively (Fig. 3e). The sensitivity was 0.667 [0.289-1.000], 0.800 [0.449-1.000], 0.800 [0.449-1.000] and 0.842 [0.678-1.000] for stage I-IV, respectively (Table 3 ). The distributions of CRC risk probability generated from the model in different stage groups were shown in Fig. 3f and demonstrated a stepwise increasing trend.

\section{Discussion}

CRC is a leading cause of cancer-related deaths worldwide. Patient non-compliance and the compromised performance of FIT-based screening strategies limit their utility in current CRC screening market. The strategy of an easily administered blood-based screening test for the early detection of CRC followed by colonoscopy validation has the potential to become an effective practice for reducing the mortality of this disease. Here, we reported the development of a novel blood-based model using four CRCspecific DNA methylation markers, c9orf50, kcnj12, znf132 and $t w i s t 1$, and demonstrated its potential for the detection of CRC in routine physical examinations.

At present, sept 9 methylation test, provided by a commercial name Epi proColon, was the only blood-based epigenetic test approved by FDA and National Medical Products Administration (NMPA, China) for CRC screening. However, its sensitivity is rather limited: one clinical trial reported that the sensitivities of Epi proColon for detecting stages I-IV CRC were 0.350, 0.630, 0.460, and 0.774 , respectively [13]. Another large cohort study of 1,544 plasma samples showed the test had a sensitivity of 0.682 and a specificity of 0.782 [15]. Another widely used biomarker CEA also showed a limited sensitivity ranging from 0.080 to 0.466 depending on the CRC stages [18]. Even though studies have shown a combination of methylated sept 9 methylation with CEA could significantly increase the sensitivity of CRC detection, the specificity was decreased significantly [16]. Therefore, the potential best test should be the one that best balances sensitivity and specificity.

In this study, we developed a new blood-based early CRC screening test, which combined two previously reported methylation markers, c9orf50 and twist1, and two novel genes, kcnj12 and $z$ f 132 . Methylated twist 1 and c9orf50 have been studied as novel blood-based markers for CRC detection recently $[17,19,20]$. Their diagnostic performances have been validated in both fecal and serum/plasma samples. kcnj12 belongs to the 
(See figure on next page.)

Fig. 3 4-marker model performance in CRC diagnosis. A representative receiver operating curve (ROC) displays the classification performance of the 4-marker model. a In the training set, the area under the curve (AUC) was 0.912. b In the test set, the AUC was 0.907. c In the validation set with advanced adenomas, the AUC was 0.796. $\mathbf{d}$ In the validation set without advanced adenomas, the AUC was 0.911. e The performance of the 4-marker model in detecting different stage CRC. HGIN, AUC 0.614. Stage I CRC, AUC 0.868. Stage II CRC, AUC 0.800. Stage III CRC, AUC 0.935. Stage IV CRC, AUC 0.947. f The risk score of the 4-marker model in detecting different stage CRC

inward-rectifier potassium channel family, which plays an important role in regulating $\mathrm{K}^{+}$channels and one study suggests that it may induce the growth of cancer cells by regulating RelA-activated NF- $\mathrm{KB}$ signaling [21]. The overexpression of ion channels and NF- $\mathrm{kB}$ signaling have been reported in multiple cancers, including colon cancer [22, 23]. However, there is limited reports demonstrating kcnj12 mutation or aberrant expression in colon cancer. As for $z$ nf132, methylation mediated silencing events have been validated in esophageal squamous cell carcinoma and breast cancer, however, not in colon cancer $[24,25]$.

Compared with methylated sept 9 methylation test, our model achieved a superior performance in detecting CRC with an overall sensitivity of 0.800 and specificity of 0.971 , comparable to the FIT test with a sensitivity of 0.790 and specificity of 0.940 . The model also had relative high sensitivities, 0.667 and 0.800 , in stage I and II $\mathrm{CRC}$, respectively. This suggests that the 4-marker panel has the potential as a screening test for CRC. The model could also be used to supplement existing FIT-based CRC screenings, e.g., as an option for the patients that refuse using the FIT test, or for triaging FIT-positives to colonoscopy to reduce the number of colonoscopies needed.

Our study has a few limitations. Firstly, the 4-marker model shows a limited diagnosis performance on the advanced adenoma plasma samples (sensitivity 0.455 and specificity 0.971). Even though the 4-marker model has a less optimized performance on advanced adenoma compared to other CRC stages, the overall sensitivity and specificity are still superior to or comparable with current established methods, e.g. blood-based mSEPT9 (sensitivity 0.171 and specificity 0.945 ) [26] and CEA (sensitivity 0.08 and specificity 0.700 ) [27], stool-based FOBT (sensitivity 0.122 and specificity 0.919 ) [26] and FIT (sensitivity 0.400 and specificity 0.940 ) [28], as well as a few published non-invasive CRC marker studies to date [29-34]. Theoretically, the diagnosis performance for advanced adenoma could further be improved by integrating other complementary markers with high sensitivity and low specificity, to develop into a multi-omics assay [32]. A larger study with more adenoma samples is needed to further examine and optimize the model by adding new markers to increase the sensitivity for detecting adenomas, which is critical to reduce CRC incidence. Secondly, we enrolled CRC patients with known cancers, most of whom were diagnosed on the basis of symptoms. Therefore, the late-stage tumor ratio within these patients would be higher than those asymptomatic individuals in a routine screening scenario. Consequently, the sensitivity of detection in a screening population might be less than that reported here. Thirdly, there is a possibility that the markers might also become positive in the plasma of some other cancer types. Therefore, future studies are needed to investigate the model's specificity against other cancer types.

In summary, we have screened 10 candidate methylation markers in the blood and developed a sensitive and specific 4-marker model for CRC early diagnosis. Once validated in a larger and asymptomatic clinical setting, this model could provide a new non-invasive choice for early tumor detection and interception in CRC.

\section{Methods}

\section{Study population}

Total 243 patients and healthy individuals with informed consent were enrolled in Sixth Affiliated Hospital of Sun Yat-sen University from 2016 to 2019, and total 29 healthy individuals were enrolled in Shenzhen People's Hospital in 2021.

Blood samples were collected in the hospital admission day or 1 to 8 days prior to surgery using EDTA Tubes (BD, Cat\# 367525). Plasma was separated within $2 \mathrm{~h}$ after receiving the whole blood samples using a standard protocol and stored at $-80^{\circ} \mathrm{C}$ until use. The plasma volume ranged from 3.0 to $5.0 \mathrm{ml}$. Repeated freezing and thawing of plasma were avoided to prevent cfDNA degradation and genomic DNA contamination from white blood cells (WBC). The normal cohort included the hospitalized patients who had physical examination or suffered from haemorrhoids, anal fissure or constipation, but without any benign, precancerous or cancerous lesions confirmed by colonoscopy.

The human methylation $450 \mathrm{~K}$ array data set and clinical characteristics of colon adenocarcinoma (COAD) and rectum adenocarcinoma (READ) were obtained from The Cancer Genome Atlas (TCGA).

\section{cfDNA extraction, bisulfite treatment and methylation analysis}

Cell-free DNA (cfDNA) was isolated by the MagMAX ${ }^{\mathrm{TM}}$ Cell-Free DNA Isolation Kit (Thermo Fisher Scientific, 

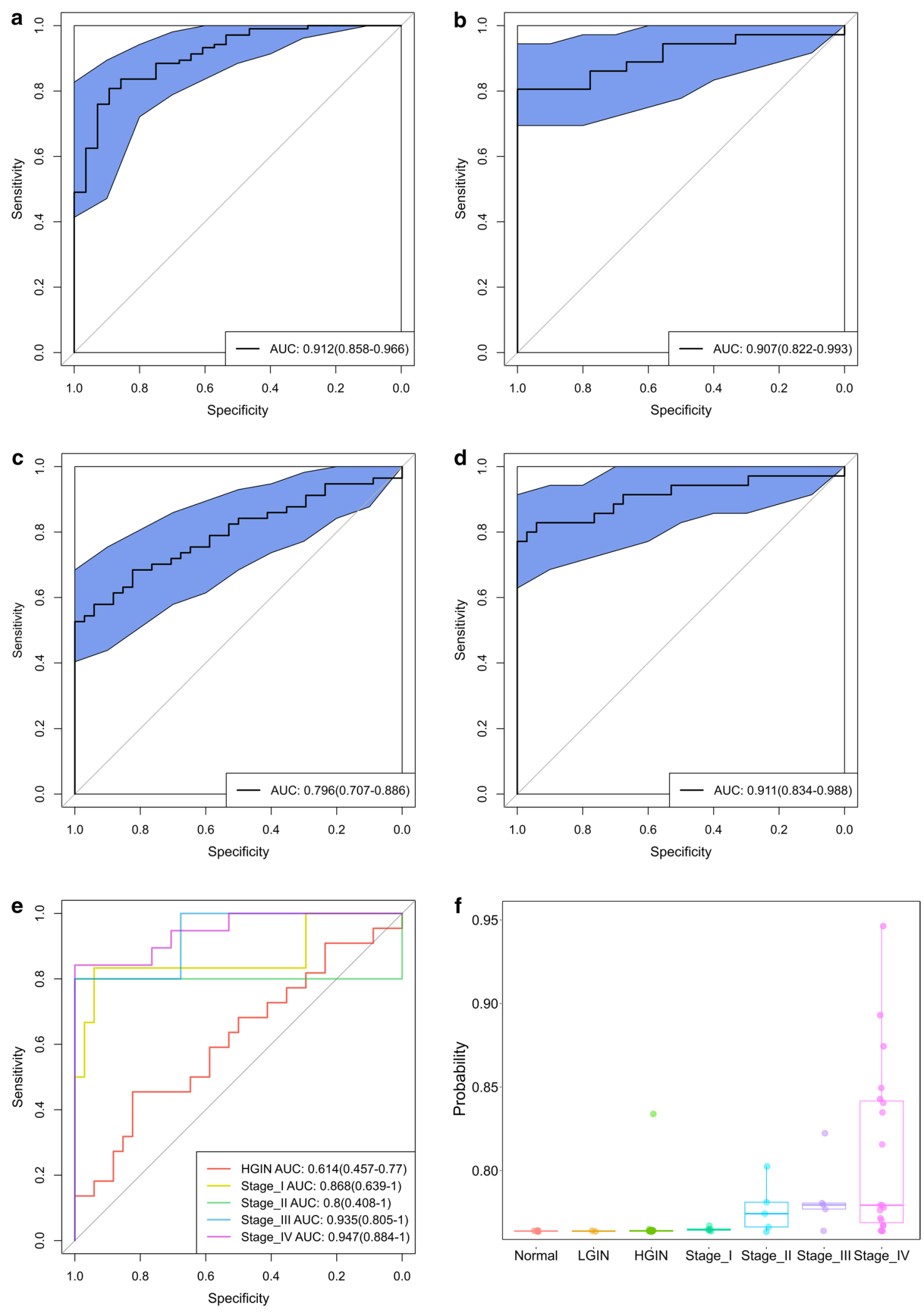
Table 3 The 4-marker model performance metrics

\begin{tabular}{|c|c|c|c|c|c|c|c|c|}
\hline & & Sensitivity & & Specificity & & Sensitivity & & \\
\hline & Overall & All_rmHGIN* & Overall & HGIN & Stage_I & Stage_II & Stage_III & Stage_IV \\
\hline Train & $\begin{array}{l}0.808(0.732- \\
0.883)\end{array}$ & $\begin{array}{l}0.808(0.732- \\
0.883)\end{array}$ & $\begin{array}{l}0.893(0.778- \\
1.000)\end{array}$ & - & $\begin{array}{l}0.786(0.571- \\
1.000)\end{array}$ & $\begin{array}{l}0.706(0.553- \\
0.859)\end{array}$ & $\begin{array}{l}0.818(0.59- \\
1.000)\end{array}$ & $\begin{array}{l}0.889(0.797- \\
0.981\end{array}$ \\
\hline Test & $\begin{array}{l}0.806(0.676- \\
0.935)\end{array}$ & $\begin{array}{l}0.806(0.676- \\
0.935)\end{array}$ & $\begin{array}{l}0.889(0.684- \\
1.000)\end{array}$ & - & $\begin{array}{l}0.833(0.535- \\
1.000)\end{array}$ & $\begin{array}{l}0.600(0.171- \\
1.000)\end{array}$ & $\begin{array}{l}0.800(0.449- \\
1.000)\end{array}$ & $\begin{array}{l}0.85(0.694- \\
1.000)\end{array}$ \\
\hline Validation & $\begin{array}{l}0.544(0.415- \\
0.673)\end{array}$ & $\begin{array}{l}0.800(0.667- \\
0.933)\end{array}$ & $\begin{array}{l}0.971(0.914- \\
1.000)\end{array}$ & $\begin{array}{l}0.455(0.227- \\
0.682)\end{array}$ & $\begin{array}{l}0.667(0.289- \\
1.000)\end{array}$ & $\begin{array}{l}0.800(0.449- \\
1.000)\end{array}$ & $\begin{array}{l}0.800(0.449- \\
1.000)\end{array}$ & $\begin{array}{l}0.842(0.678- \\
1.000)\end{array}$ \\
\hline
\end{tabular}

*Removed HGIN (AA) samples

Cat\# A29319) according to the manufacture's protocol. The concentration of cfDNA was measured by Qubit ${ }^{\mathrm{TM}}$ dsDNA HS Assay Kit (Thermo Fisher Scientific, Cat\# Q32854) and the quality was examined using the Agilent High Sensitivity DNA Kit (Cat\# 5067-4626) on a Agilent 2100 Bioanalyzer Instrument. $10 \mathrm{ng}$ of cfDNA without overly genomic DNA contamination were proceeded to perform the following assays.

Bisulfite conversion was performed using the Zymo EZ DNA Methylation-Direct Kit (Zymo Research, Cat\# D5044) according to the manufacturer's protocol. Briefly, cfDNA was first denatured at $98{ }^{\circ} \mathrm{C}$ for $10 \mathrm{~min}$, then treated with sodium bisulfite at $64{ }^{\circ} \mathrm{C}$ for $3.5 \mathrm{~h}$. Then, DNA was added into a spin column and desulphonated by adding desulphonation buffer and incubated at room temperature for $20 \mathrm{~min}$. Bisulfite-converted DNA was purified by a spin column and eluted with $25 \mu \mathrm{L}$ of Elution Buffer.

The bisulfite-modified DNA were further analyzed by a CRC 10-Gene Meth-Detect Kit according to the manufacturer's instruction (AnchorDx, China, Cat\# CRCB012). In short, we used a strategy of multiplexing PCRs followed by the quantitative methylation-specific PCR (qMSP) quantification for each candidate methylation marker to overcome the limited quantity of cfDNA in detecting ten methylation markers simultaneously (Additional file 1: Figure S1), as previously reported [35, 36]. A multiplex 10-marker targeted pre-amplification of methylated regions was performed on $15 \mu \mathrm{l}$ of the bisulfite-treated DNA using $25 \mu$ l of the Meth-Pre-Amp Master Mix (AnchorDx, China, Cat\# CRCB012-01) and $10 \mu$ of Meth-Pre-Amp 10-Gene CRC Panel (AnchorDx, China, Cat\# CRCB012-02, primer sequence shown in Additional file 1: Table S1) in the kit in a thermal cycler (Thermo Fisher, United States, Cat\# 4484073) under the following cycling conditions: denaturation at $98{ }^{\circ} \mathrm{C}$ for $30 \mathrm{~s}, 5$ cycles $\left(98^{\circ} \mathrm{C}\right.$ for $15 \mathrm{~s}, 58^{\circ} \mathrm{C}$ for $15 \mathrm{~s}$ and $72{ }^{\circ} \mathrm{C}$ for $15 \mathrm{~s}), 17$ cycles $\left(98^{\circ} \mathrm{C}\right.$ for $15 \mathrm{~s}, 63{ }^{\circ} \mathrm{C}$ for $15 \mathrm{~s}$ and $72{ }^{\circ} \mathrm{C}$ for $15 \mathrm{~s}$ ), final extension at $72{ }^{\circ} \mathrm{C}$ for $5 \mathrm{~min}$ and hold at $4{ }^{\circ} \mathrm{C}$.
The amplified products were diluted $2 \mathrm{X}$ before used as template for quantitative methylation-specific PCR on the QuantStudio 3 Real-Time PCR System (Thermo Fisher, United States) using Meth-Quant Master Mix (AnchorDx, China, Cat\# CRCB012-03) and 10-Gene CRC Detect Panel (AnchorDx, China, Cat\# CRCB01204, primer/probe sequence shown in Additional file 1: Table S1) under the following cycling conditions: denaturation at $98{ }^{\circ} \mathrm{C}$ for $30 \mathrm{~s}, 40$ cycles $\left(95^{\circ} \mathrm{C}\right.$ for $10 \mathrm{~s}, 62^{\circ} \mathrm{C}$ for $20 \mathrm{~s}$ ), with fluorescent signal collected at the annealing/extension step $\left(62^{\circ} \mathrm{C}\right.$ for $\left.40 \mathrm{~s}\right)$.

\section{Modeling and statistical analysis}

The percentage methylated reference (PMR) was calculated by dividing the dividing the normalized quantity (target gene/reference gene ratio) of the samples by the normalized quantity of the methylation positive controls and multiplying by 100 [37]. Briefly, a standard curve between $\Delta \mathrm{Ct}$ and methylation percentage was established for each marker using different methylation standards $(0 \%, 0.1 \%, 0.33 \%, 1 \%, 3.3 \%, 10 \%, 33 \%, 66 \%$ and $100 \%$ in current study). PMR was calculated based on the function of $\Delta \mathrm{Ct}$ and methylation percentage obtained from the standard curve. ACTB was used as a default reference for normalization.

The logistic regression-based models were constructed using Python Sklearn packages. For a single gene marker, we used the target gene and the reference gene ACTB for fitting. The optimal threshold was determined by Youden's index. For the model development, we randomly divided the whole cohort into two parts, the model development set $(80 \%)$ and the validation set $(20 \%)$. The model development set was used for model building and testing while the validation set for evaluating the model performance. The performance of each model was evaluated by area under receiver operating characteristic curve (AUC) using the R package pROC.

We used Fisher exact statistic test for categorical variables including gender and Wilcoxon test for continuous variables like age. All statistical analysis was performed 
with R software, version 3.32. Unless otherwise specified, all statistical tests were two-sided.

\section{Abbreviations}

AUC: Area under curve; CRC: Colorectal cancer; HP: Hyperplastic polys; NAA: Non-advanced adenomas; AA: Advanced adenomas; PMR: Percentage of methylated reference.

\section{Supplementary Information}

The online version contains supplementary material available at https://doi. org/10.1186/s13148-021-01076-8.

Additional file 1. Supplemental Figures and Tables.

\section{Acknowledgements}

We thank all the participants for their generosity.

\section{Authors' contributions}

Z.C., X.W. and J.B.F. conceived the study. Y.Z., Q.W., Z.C. and L.X. designed the experiments. Y.Z. and Q.W. performed the patient enrollment and sample collection. Y.Z., Q.W., L.X., S.L. and Y.L. performed the experiments. H.W. and T.H. performed the data modelling. All authors contributed to data acquisitions. X.L. and H.W. contributed to data visualization. Y.Z. and X.L. Wrote the original draft of the paper. Z.C., X.W. and J.B.F. contributed substantially to manuscript versions. All authors read and approved the manuscript.

\section{Funding}

Science and Technology Planning Project of Guangdong Province, China(Grant NO.2017B020226005); Scheme of Guangzhou for Leading Team in Innovation(Grant NO.201909010010); Scheme of Guangzhou for Leading Talents in Innovation and Entrepreneurship(Grant NO.2016007); Scheme of Guangzhou Economic and Technological Development District for Leading Talents in Innovation and Entrepreneurship (Grant NO.2017-L152); National Key R\&D Program of China (No. 2017YFC1308800); National Natural Science Foundation of China (No. 81870383); Clinical Innovation Research Program of Bioland Laboratory (Guangzhou Regenerative Medicine and Health Guangdong Laboratory) (No. 2018GZR0201005); Science and Technology Planning Project of Guangzhou City (No. 201804010014, 201803040019) and Sun Yatsen University 5010 Project (N0.2010012).

\section{Availability of data and materials}

The datasets used and/or analyzed during the current study are available from the corresponding author on reasonable request.

\section{Declarations}

\section{Ethics approval and consent to participate}

Ethics approvals were granted by the ethics committee of The Sixth Affiliated Hospital of Sun Yat-sen University and Shenzhen People's Hospital. The written informed consent was obtained from all participants.

\section{Competing interests}

L.X., H.W., X.L., S.L., T.H., Y.L., Z.C. and J.B.F. are employees of AnchorDx Medical Co., Ltd. or AnchorDx, Inc. Other authors declare no competing financial interest.

\section{Author details}

${ }^{1}$ Department of Colorectal Surgery, The Sixth Affiliated Hospital, Sun Yat-Sen University, Guangzhou 510655, Guangdong, China. ${ }^{2}$ Guangdong Provincial Key Laboratory of Colorectal and Pelvic Floor Diseases, The Sixth Affiliated Hospital, Sun Yat-Sen University, Guangzhou 510655, Guangdong, China. ${ }^{3}$ AnchorDx Medical Co., Ltd, Unit 502, 3rd Luoxuan Road, International Bio-Island, Guangzhou 510300, China. ${ }^{4}$ AnchorDx Inc., 6305 Landing Pkwy, Fremont, CA 94538, USA. ${ }^{5}$ Department of Pathology, School of Basic Medical Science, Southern Medical University, Guangzhou 510515, China.
Received: 4 February 2021 Accepted: 13 April 2021

Published online: 23 April 2021

\section{References}

1. Siegel RL, Miller KD, Goding Sauer A, Fedewa SA, Butterly LF, Anderson JC, et al. Colorectal cancer statistics, 2020. CA Cancer J Clin. 2020;70(3):145-64.

2. Rex DK, Boland CR, Dominitz JA, Giardiello FM, Johnson DA, Kaltenbach $T$, et al. Colorectal cancer screening: recommendations for physicians and patients from the US Multi-Society Task Force on Colorectal Cancer Gastroenterology. Gastroenterology. 2017;153(1):307-23.

3. Wolf AM, Fontham ET, Church TR, Flowers CR, Guerra CE, LaMonte SJ, et al. Colorectal cancer screening for average-risk adults: 2018 guideline update from the American Cancer Society. CA Cancer J Clin. 2018;68(4):250-81.

4. Vijan S, Hwang EW, Hofer TP, Hayward RA. Which colon cancer screening test? A comparison of costs, effectiveness, and compliance. Am J Med. 2001;111(8):593-601.

5. Berger BM, Schroy PC III, Dinh TA. Screening for colorectal cancer using a multitarget stool DNA test: modeling the effect of the intertest interval on clinical effectiveness. Clin Colorectal Cancer. 2016;15(3):e65-74.

6. Osborne J, Wilson C, Moore V, Gregory T, Flight I, Young G. Sample preference for colorectal cancer screening tests: Blood or stool? 2012.

7. Janson W, Jenkins LJ, Chionh F, Mariadason JM. Aberrant DNA methylation in colorectal cancer: what should we target? Trends Cancer. 2017:3(10):698-712.

8. Bennett RL, Licht JD. Targeting epigenetics in cancer. Annu Rev Pharmacol Toxicol. 2018;58:187-207.

9. Okugawa Y, Grady WM, Goel AJG. Epigenetic alterations in colorectal cancer: emerging biomarkers. Gastroenterology. 2015;149(5):1204-25.

10. Verma M, Kumar VJ. Epigenetic biomarkers in colorectal cancer. Mo Diagn Ther. 2017;21(2):153-65.

11. Danese E, Montagnana MJ. Epigenetics of colorectal cancer: emerging circulating diagnostic and prognostic biomarkers. Ann Transl Med. 2017;5(13):279.

12. Payne SRJE. From discovery to the clinic: the novel DNA methylation biomarker $m$ SEPT9 for the detection of colorectal cancer in blood. Epigenomics. 2010;2(4):575-85.

13. Xie L, Jiang X, Li Q, Sun Z, Quan W, Duan Y, et al. Diagnostic value of methylated Septin9 for colorectal cancer detection. Front Oncol. 2018;8:247.

14. Yan S, Liu Z, Yu S, Bao YJ. Diagnostic value of methylated septin9 for colorectal cancer screening: a meta-analysis. Med Sci Monit. 2016:22:3409.

15. Sun G, Meng J, Duan H, Zhang D, Tang YJ. Diagnostic assessment of septin9 DNA methylation for colorectal cancer using blood detection: a meta-analysis. Pathol Oncol Res. 2019;25(4):1525-34.

16. Ma ZY, Law WL, Ng EKO, Chan CSY, Lau KS, Cheng YY, et al. Methylated Septin 9 and carcinoembryonic antigen for serological diagnosis and monitoring of patients with colorectal cancer after surgery. Sci Rep. 2019;9(1):1-9.

17. Zhang X, Wan S, Yu Y, Ruan W, Wang H, Xu L, et al. Identifying potential DNA methylation markers in early-stage colorectal Cancer. Genomics. 2020;112(5):3365-73.

18. Hundt S, Haug U, Brenner HJCE, Biomarkers P. Blood markers for early detection of colorectal cancer: a systematic review. Cancer Epidemiol Biomark Prev. 2007;16(10):1935-53.

19. Okada T, Suehiro Y, Ueno K, Mitomori S, Kaneko S, Nishioka M, et al. TWIST1 hypermethylation is observed frequently in colorectal tumors and its overexpression is associated with unfavorable outcomes in patients with colorectal cancer. Genes. 2010;49(5):452-62.

20. Jensen $S \varnothing, \varnothing$ gaard N, Ørntoft MBW, Rasmussen MH, Bramsen JB, Kristensen $\mathrm{H}$, et al. Novel DNA methylation biomarkers show high sensitivity and specificity for blood-based detection of colorectal cancer-a clinical biomarker discovery and validation study. Clin Epigenet. 2019;11(1):1-14.

21. Lee I, Lee S-J, Kang TM, Kang WK, Park CJ. Unconventional role of the inwardly rectifying potassium channel Kir2.2 as a constitutive activator of RelA in cancer. Cancer Res. 2013;73(3):1056-62.

22. Lastraioli E, lorio J, Arcangeli AJ. Ion channel expression as promising cancer biomarker. Biochimica et Biophysica Acta (BBA) Biomembranes. 2015;1848(10):2685-702. 
23. Dolcet X, Llobet D, Pallares J, Matias-Guiu XJ. NF-kB in development and progression of human cancer. Virchows Arch. 2005;446(5):475-82.

24. Jiang D, He Z, Wang C, Zhou Y, Li F, Pu W, et al. Epigenetic silencing of ZNF132 mediated by methylation-sensitive Sp1 binding promotes cancer progression in esophageal squamous cell carcinoma. Cell Death Dis. 2018;10(1):1-13.

25. Liu J, Liu R, Xue M, Zhang W, Zhao X, Xia P. Downregulated ZNF132 predicts unfavorable outcomes in breast cancer via hypermethylation modification. BMC Cancer. 2021;21:1-12

26. Sun J, Fei F, Zhang M, Li Y, Zhang X, Zhu S, et al. The role of $m$ SEPT9 in screening, diagnosis, and recurrence monitoring of colorectal cancer. BMC Cancer. 2019;19(1):1-10.

27. Gao Y, Wang J, Zhou Y, Sheng S, Qian SY, Huo XJ. Evaluation of serum CEA, CA19-9, CA72-4, CA125 and ferritin as diagnostic markers and factors of clinical parameters for colorectal cancer. Sci Rep. 2018;8(1):1-9.

28. Lee JK, Liles EG, Bent S, Levin TR, Corley DA. Accuracy of fecal immunochemical tests for colorectal cancer: systematic review and meta-analysis. Ann Intern Med. 2014;160(3):171-81.

29. Rho J-H, Ladd JJ, Li Cl, Potter JD, Zhang Y, Shelley D, et al. Protein and glycomic plasma markers for early detection of adenoma and colon cancer. Gut. 2018;67(3):473-84.

30. Jeun M, Lee HJ, Park S, Do EJ, Choi J, Sung YN, et al. A novel blood-based colorectal cancer diagnostic technology using electrical detection of colon cancer secreted protein-2. Adv Sci. 2019;6(11):1802115.

31. Werner S, Krause F, Rolny V, Strobl M, Morgenstern D, Datz C, et al. Evaluation of a 5-marker blood test for colorectal cancer early detection in a colorectal cancer screening setting. Clin Cancer Res. 2016;22(7):1725-33.
32. Xue VW, Cheung MT, Chan PT, Luk LL, Lee VH, Au TC, et al. Non-invasive potential circulating mRNA markers for colorectal adenoma using targeted sequencing. Sci Rep. 2019;9(1):1-10.

33. Zhao G, Li H, Yang Z, Wang Z, Xu M, Xiong S, et al. Multiplex methylated DNA testing in plasma with high sensitivity and specificity for colorectal cancer screening. Cancer Med. 2019;8(12):5619-28.

34. Pickhardt PJ. Emerging stool-based and blood-based non-invasive DNA tests for colorectal cancer screening: the importance of cancer prevention in addition to cancer detection. Abdom Radiol NY. 2016;41(8):1441-4.

35. Zeka F, Vanderheyden K, De Smet E, Cuvelier CA, Mestdagh P, Vandesompele JJ. Straightforward and sensitive RT-qPCR based gene expression analysis of FFPE samples. Sci Rep. 2016;6(1):1-10.

36. Kibschull M, Lye SJ, Okino ST, Sarras HJ. Quantitative large scale gene expression profiling from human stem cell culture micro samples using multiplex pre-amplification. Syst Biol Reprod Med. 2016;62(1):84-91.

37. Eads CA, Lord RV, Wickramasinghe K, Long TI, Kurumboor SK, Bernstein L, et al. Epigenetic patterns in the progression of esophageal adenocarcinoma. Cancer Res. 2001;61(8):3410-8.

\section{Publisher's Note}

Springer Nature remains neutral with regard to jurisdictional claims in published maps and institutional affiliations.
Ready to submit your research? Choose BMC and benefit from:

- fast, convenient online submission

- thorough peer review by experienced researchers in your field

- rapid publication on acceptance

- support for research data, including large and complex data types

- gold Open Access which fosters wider collaboration and increased citations

- maximum visibility for your research: over $100 \mathrm{M}$ website views per year

At BMC, research is always in progress.

Learn more biomedcentral.com/submissions 